\title{
Compensation of Backlash for Geared Drive Systems and Thrust Wires Used in Teleoperation
}

\author{
D Kasun Prasanga ${ }^{* a)}$ Non-member, Emre Sariyildiz ${ }^{* *}$ Non-member \\ Kouhei Ohnishi*** Fellow
}

(Manuscript received Feb. 21, 2014, revised March 5, 2015)

\begin{abstract}
This paper presents a unique method to cancel the backlash present in geared drive systems and thrust-wire systems. Large-sized robots need larger actuators with a high inertia to obtain the desired amount of torque. However, the use of large actuators will increase the effect of gravity. To compensate for the gravitational effect, motors with a high torque are again necessary. Therefore, geared drive systems are used to obtain higher torques and decrease the actuator size. However, adding gears will create a backlash problem. Moreover, when it is necessary to make lighter robots, thrust wires can be used to transmit the motion from the motor to the end effector while keeping them separated. However, the mechanical backlash inside the thrust wire is again a matter of concern. If geared systems and thrust-wire systems are used for the transmission of haptic information in bilaterally controlled systems, the backlash will greatly deteriorate the performance of the system and necessitate compensation. Therefore, a novel method is proposed to uniquely cancel the backlash in the gears and thrust wires used in bilateral teleoperation with twist control. This method uses two identical counter-operating drives per joint to compensate for the backlash effect with a unique control structure that can be used in both geared systems and thrust-wire systems. Experiments have been carried out for the proposed method to validate its accuracy.
\end{abstract}

Keywords: backlash compensation, haptics, bilateral control, geared drives, thrust wires, teleoperation

\section{Introduction}

There are so many industries in the modern world, that get the support of the power assisted drives and systems ${ }^{(1)}$. Many of those industries are supported by robots to handle their major tasks. To obtain higher torques for the robots, motors having larger inertias are necessary to be used in general. Due to the impracticality involved in it, geared drives are used to achieve the required torque with small comparatively motors ${ }^{(2)(3)}$. However, adding gears will introduce the backlash problem. If it is desired to obtain lighter weight robot designs, and improve the stability of force control by decreasing the inertia, thrust wires can be used to keep the actuator and the end effector apart ${ }^{(4)(5)}$. Similar to the spacing between the meshing teeth create backlash in gears, the spacing between inner wire and outer tube creates backlash in thrust wires. If the robot system is used to transmit the haptic information, backlash will become problematic in both geared systems and thrust wired systems. Therefore compensation for backlash is necessary.

a) Correspondence to: D Kasun Prasanga. E-mail: prasanga@ ieee.org

${ }^{*}$ School of Integrated Design Engineering, Keio University 3-14-1, Hiyoshi, Kohoku-ku, Yokohama 223-8522, Japan

${ }^{* *}$ Department of Biomedical Engineering, National University of Singapore Singapore

*** Department of System Design Engineering, Keio University 3-14-1, Hiyoshi, Kohoku-ku, Yokohama 223-8522, Japan
When considering the gear backlash compensation, some attempts have been made by various researchers to achieve it. Yi Ling et al. ${ }^{\left({ }^{(6)}\right.}$ and Chao He et al. ${ }^{(7)}$ have attempted to compensate the backlash by different modeling methods, however their approaches mainly depend on the accuracy of the modeling. Sensor-less backlash compensation by considering the impulse generated at the point of meshing to determine the instance of gear meshing has also been studied ${ }^{(8)(9)}$. However this may result in some undesired decisions when the impulse is generated from the load side. Kolnik et al. ${ }^{(10)}$ and several other researchers ${ }^{(11)-(15)}$ have attempted to make a compensation to the gear backlash by treating it as torque disturbance, but a complete elimination of backlash has not yet been realized. Control methods with the use of multiple drive units have been used by some researchers to compensate the backlash ${ }^{(16)(17)}$. How ever the presence of several redundant motors will eventually make the system bulky making it unsuitable for the applications related to haptics. Simulation based approach to treat the backlash has also been attempted by some other researchers, however in their approach, the friction inside the system is increased ${ }^{(18)}$. Several other approaches made in the literature for compensation of backlash have not been able to completely eliminate the backlash problem ${ }^{(19)-(21)}$. To overcome this problem mechanically, a special type of gears called zero backlash gears and harmonic drive systems have been introduced. However adding a zero backlash gear cannot compensate the entire backlash presented in a geared system as it can cancel the backlash of the immediate meshing gears, and a harmonic drive will 
create low stiffness and non-linear friction effects ${ }^{(22)}$. When considering the thrust wire backlash, the construction of the wire has accounted for its backlash problem. There is a space between the inner wire and the outer tube of a thrust wire. This gap allows the inner wire to bend inside the outer tube when applied with a sufficient force, and will ultimately resulted in a backlash between the two endpoints of the thrust wire. Therefore the motion transmission is affected by the spacing inside the wire ${ }^{(23)}$. Recently, there have been several attempts to compensate the backlash effect ${ }^{(24)(25)}$ in thrust wires. However these attempts either use additional encoder or force sensor at the end of the thrust wire or at the end effector, making it bulky for the light weighted robots where necessary. Therefore a compensation for the backlash is essential for both geared drives and thrust wired systems.

The contribution of this paper is to propose a novel method to realize backlash cancellation of gears and thrust wires by using a unique control structure. The motivation of this research is to realize a backlash free motion transmission in a bilaterally controlled system. This proposed method can uniquely solve the backlash problem for both geared motor drives and thrust wired systems. Therefore, the proposed method can be especially used in the bilateral teleoperation systems where backlash free motion transmission is essential. This method does not use any additional encoder or force sensor at the end effector. Instead, two identical counter operating drives are used. The proposed control structure can keep the geared system or the thrust wired system interlocked with each other to eliminate the backlash and operate in a load sharing basis depend on the direction of operation. In the experiments, master systems and slave systems are used and they are controlled bilaterally. A direct drive was chosen for the master robot that doesn't contain the backlash. For the slave side, two counter operating drives each having backlash are used. Using the proposed control structure, the backlash of the slave side is compensated and it was shown that the proposed method works well. The haptic transmission was taken place between the master robot and the end effector of the slave side through the bilateral control.

This paper is organized in the following manner. In the Sect. 2, system modeling is explained. Acceleration based control, four channel bilateral control together with disturbance observer, and system control are explained in Sect. 3. Experimental results obtained for the gear backlash cancellation and thrust wire backlash cancellation together with discussion are shown in Sect. 4. This paper concludes in Sect. 5.

\section{Modeling}

2.1 Gear Backlash Gear backlash is the presence of space between the teeth of two meshing gears. This space occurs due to the imperfect meshing between the gear wheels. This phenomenon is shown in Fig. 1. Here, one side of tooth $\mathrm{Q}$ is in contact with tooth $\mathrm{P}$, however the other side of the tooth $\mathrm{Q}$ is not in contact with the tooth $\mathrm{R}$ of gear 1 . Therefore there exist a space between the tooth $\mathrm{Q}$ and tooth $\mathrm{R}$ and it is called as the gear backlash. This makes a problem of transferring the position and torque if the velocity of either driving wheel or driven wheel is changed.

2.2 Thrust Wire Backlash Thrust wire consists of an outer tube and an inner wire. The cross section of the thrust

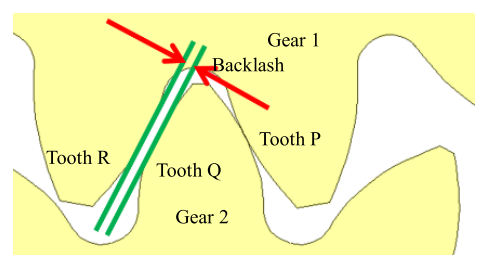

Fig. 1. Gear backlash

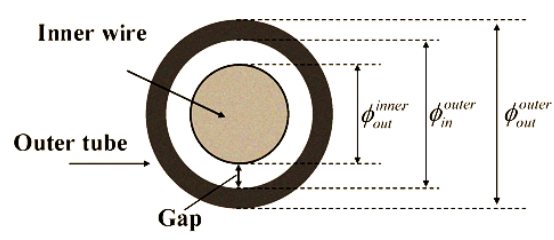

Fig. 2. Cross section of the thrust wire

Table 1. Specification of the thrust wire used for the experiment

\begin{tabular}{|c|c|c|c|c|}
\hline \multicolumn{2}{|c|}{ Outer tube } & \multirow{2}{*}{$\begin{array}{c}\text { Inner wire } \\
\emptyset_{\text {out }}^{\text {inner }} \\
{[\mathrm{mm}]}\end{array}$} & \multirow{2}{*}{$\begin{array}{l}\text { Gap [mm] } \\
\emptyset_{\text {in }}^{\text {outer }} \\
-\emptyset_{\text {out }}^{\text {inner }}\end{array}$} & \multirow[b]{2}{*}{$\begin{array}{c}\text { Length } \\
{[\mathrm{mm}]}\end{array}$} \\
\hline $\begin{array}{l}\emptyset_{\text {out }}^{\text {outer }} \\
{[\mathrm{mm}]}\end{array}$ & $\begin{array}{c}\emptyset_{\text {in }}^{\text {outer }} \\
{[\mathrm{mm}]}\end{array}$ & & & \\
\hline 3.0 & 1.4 & 1.2 & 0.2 & 1500.0 \\
\hline
\end{tabular}

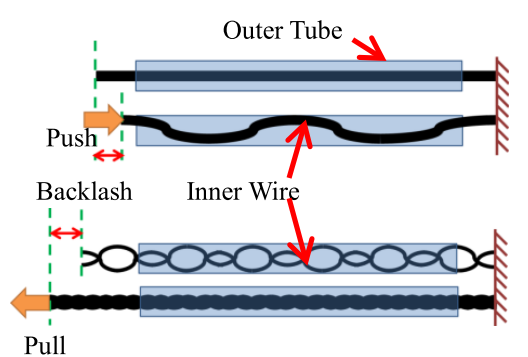

Fig. 3. Backlash due to pushing and pulling

wire used is shown in Fig. 2 and the measured dimensions of the wire are listed in Table 1. There is an air gap exists between the inner wire and the outer tube of a thrust wire. The outer tube guides the inner wire, so that both pushing and pulling motion for the inner wire can be realized.

There are several reasons for the backlash to occur inside a thrust wire. One reason is the clearance between the inner wire and the inner wall of the outer tube. This allows the inner wire to bend inside the outer tube when the wire is pushed. Since the inner wire consists of twisting several strands, the length of the inner wire is slightly shorter than that of it when the wire is pulled by applying a tension. Also, wire strands become more loosen and the wire becomes shorter when the wire is pushed. This phenomenon is illustrated in the Fig. 3. If a bending of the thrust wire is concerned, the inner wire will be in different positions inside the outer tube depending on the applied force. This is shown in Fig. 4. Above mentioned problems causes the backlash of the thrust wire. When the backlash is present, it affects the transmission of the position information from one end to the other. This greatly affects if the application considered is related to the haptics or the transmission of tactile information. Especially when the thrust wire movement direction changes the presence of the backlash causes a considerable position error. Therefore a compensation for the backlash is necessary.

2.3 Proposal The proposed method can uniquely 


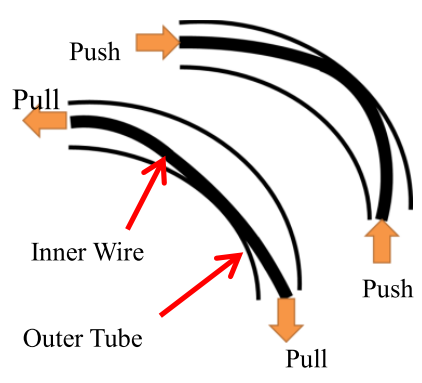

Fig. 4. Backlash when bending

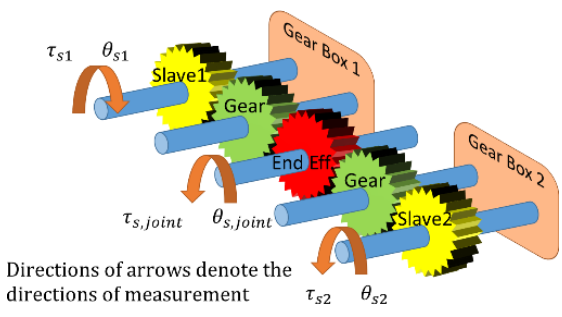

Fig. 5. Proposed solution for gear case

cancel the backlash in both gear case and the thrust wire case as it keeps the two slave drives in an interlocked state by maintaining counteracting constant torque or force, while operating in an output torque or force sharing principle.

In the gear case, a constant torque between the very first gear to the very end gear is maintained by using two motor drives to achieve the backlash cancellation. The two motors apply constant counter acting torque, named "twisting torque" over the gear system to eliminate the gaps presented between the teeth of the gear wheels. The so called Twisting torque is generated by twist control of the proposal. This enables the elimination of the complete backlash of the whole cascaded gear system. The proposed controller is designed in a way that the slave motors share the load torque of the slave side based on the direction of rotation. This can also be considered as a modified case of tension control for the tendon driven systems $^{(26)(27)}$. This idea is illustrated in Fig. 5. Here, slave 1 and slave 2 are the slave motors attached gears that are counter operating. Slave1 gear and its adjacent green colored gear represent the all the gears inside gearbox 1. Likewise, the slave 2 gear and its adjacent green colored gear represent the all the gears inside the gearbox-2. For the modeling purpose of this concept, the position measurement and the torque measurement directions were used as positive in the directions marked in the Fig. 5.

When considering the thrust wire case, the backlash of a thrust wire does not affect much for the performance, if the thrust wire is used to apply a continuous action of either pulling or pushing of an object. Considering this phenomena into account, two thrust wires of the slave actuators are laid in parallel and kept one wire tensed and the other pushed while the operation to cancel the backlash as shown in Fig. 6. Here also, two slave actuators maintain a constant force to keep the two thrust wires in an interlocked state while the force is shared according to the direction of motion similar to the gear backlash cancellation method.

Because of the similarity in operation, controlling of the geared drive system and thrust wire system are achieved by a similar control structure to uniquely cancel the backlash

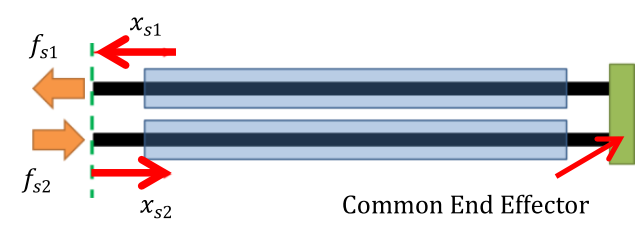

Fig. 6. Proposed solution for thrust wire case

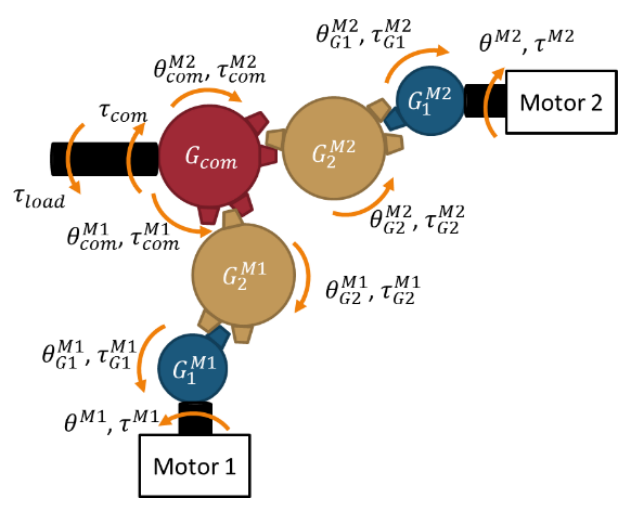

Fig. 7. Proposed method

problem of two systems.

2.4 Mathematical Modeling For the convenience, let us consider a cascaded gear system for the modeling. Since there are two motor drive systems are supporting the common load and a coupling between two motor drives is also presented, detailed diagram for analysis can be illustrated as in Fig. 7. For the purpose of clarity, most important gear tooth of the gears are only shown in the figure. Gear 1 of motor $1\left(G_{1}^{M 1}\right)$ is the gear that is directly attached to the shaft of the Motor 1. $G_{2}^{M 1}$ represents all the gears placed in between $G_{1}^{M 1}$ and the common gear $\left(G_{c o m}\right)$. The other gears represent the respective gears that belong to the motor 2 .

According to the Fig. 7, equation for the motor 1 can be written as,

$$
J_{M 1} \ddot{\theta}_{M 1}+b_{M 1} \dot{\theta}_{M 1}={ }^{M 1} \tau_{M 1}-{ }^{G 1} \tau_{G 1}^{M 1} \ldots \ldots \ldots \ldots \ldots
$$

Equations for gear 1 of motor 1 and gear 2 of motor 2 can be expressed as,

$$
\begin{aligned}
& J_{G 1}^{M 1} \ddot{\theta}_{G 1}^{M 1}+b_{G 1}^{M 1} \dot{\theta}_{G 1}^{M 1}={ }^{G 1} \tau_{G 1}^{M 1}-\frac{N_{G 1}^{M 1}}{N_{G 2}^{M 1}}{ }^{G 2} \tau_{G 2}^{M 1}, \ldots \ldots \\
& J_{G 2}^{M 1} \ddot{\theta}_{G 2}^{M 1}+b_{G 2}^{M 1} \dot{\theta}_{G 2}^{M 1}={ }^{G 2} \tau_{G 2}^{M 1}-\tau_{c o m}^{M 1} . \cdots \ldots \ldots \ldots \ldots
\end{aligned}
$$

But,

$$
\theta_{G 1}^{M 1}=\theta_{M 1} \text { and } \theta_{G 2}^{M 1}=\frac{N_{G 1}^{M 1}}{N_{G 2}^{M 1}} \theta_{M 1} \ldots \ldots \ldots \ldots \ldots \ldots
$$

Therefore, the motor 1 equation can be rewritten as,

$$
\begin{aligned}
& \left(J_{M 1}+J_{G 1}^{M 1}+\frac{N_{G 1}^{M 1}}{N_{G 2}^{M 1}} J_{G 2}\right) \ddot{\theta}_{M 1} \\
& \quad+\left(b_{M 1}+b_{G 1}^{M 1}+\frac{N_{G 1}^{M 1}}{N_{G 2}^{M 1}} J_{G 2}\right) \dot{\theta}_{M 1}={ }^{M 1} \tau_{M 1}-\beta_{1} \tau_{c o m}^{M 1},
\end{aligned}
$$

And similarly, for the motor 2,

$$
\left(J_{M 2}+J_{G 1}^{M 2}+\frac{N_{G 1}^{M 2}}{N_{G 2}^{M 2}} J_{G 2}\right) \ddot{\theta}_{M 2}
$$




$$
+\left(b_{M 2}+b_{G 1}^{M 2}+\frac{N_{G 1}^{M 2}}{N_{G 2}^{M 2}} J_{G 2}\right) \dot{\theta}_{M 2}={ }^{M 2} \tau_{M 2}-\beta_{2} \tau_{c o m}^{M 2} .
$$

Where,

$$
\beta_{1}=\frac{N_{G 1}^{M 1}}{N_{c o m}} \text { and } \beta_{2}=\frac{N_{G 1}^{M 2}}{N_{c o m}} .
$$

For the common gear,

$$
\begin{aligned}
J_{c o m} \ddot{\theta}_{c o m}+b_{\text {com }} \dot{\theta}_{\text {com }} & =\tau_{\text {com }}-\tau_{\text {load }} \\
& =\tau_{\text {com }}^{M 1}-\tau_{\text {com }}^{M 2}-\tau_{\text {load }}
\end{aligned}
$$

Here,

$$
\theta_{\text {com }}=\theta_{c o m}^{M 1}-\theta_{c o m}^{M 2}=\frac{N_{G 1}^{M 1}}{N_{c o m}} \theta_{M 1}-\frac{N_{G 1}^{M 2}}{N_{c o m}} \theta_{M 2} . \cdots \cdots
$$

For the simplicity, we can write,

$$
\begin{aligned}
& \left(J_{M 1}^{*}\right) \ddot{\theta}_{M 1}+\left(b_{M 1}^{*}\right) \dot{\theta}_{M 1}={ }^{M 1} \tau_{M 1}-\beta_{1} \tau_{c o m}^{M 1}, \ldots \\
& \left(J_{M 2}^{*}\right) \ddot{\theta}_{M 2}+\left(b_{M 2}^{*}\right) \dot{\theta}_{M 2}={ }^{M 2} \tau_{M 2}-\beta_{2} \tau_{c o m}^{M 2}, \ldots \\
& J_{c o m}\left(\beta_{1} \ddot{\theta}_{M 1}-\beta_{2} \ddot{\theta}_{M 2}\right)+b_{c o m}\left(\beta_{1} \dot{\theta}_{M 1}-\beta_{2} \dot{\theta}_{M 2}\right) \\
& \quad=\tau_{\text {com }}^{M 1}-\tau_{\text {com }}^{M 2}-\tau_{\text {load }} \ldots \ldots \ldots \ldots \ldots \ldots \ldots
\end{aligned}
$$

Take,

$$
\theta_{M 1}=\frac{N_{G 1}^{M 2}}{N_{G 2}^{M 1}} \theta_{M 2}=\beta_{3} \theta_{M 2}
$$

Therefore, we can write:

$$
\begin{aligned}
& {\left[\left(J_{\text {com }} \beta_{1}+J_{M 1}^{*} \beta_{1}^{-1}\right)-\left(J_{\text {com }} \beta_{2}+\beta_{2}^{-1} J_{M 2}^{*}\right) \beta_{3}^{-1}\right] \ddot{\theta}_{M 1}} \\
& \quad+\left[\left(b_{\text {com }} \beta_{1}+b_{M 1}^{*} \beta_{1}^{-1}\right)-\left(b_{\text {com }} \beta_{2}+\beta_{2}^{-1} b_{M 2}^{*}\right) \beta_{3}^{-1}\right] \dot{\theta}_{M 1} \\
& =\beta_{1}^{-1 M 1} \tau_{M 1}-\tau_{\text {load }} \ldots \ldots \ldots \ldots \ldots \ldots \ldots \ldots \ldots \ldots \ldots \ldots \ldots \ldots
\end{aligned}
$$

And,

$$
\begin{aligned}
& {\left[\left(J_{\text {com }} \beta_{1}+J_{M 1}^{*} \beta_{1}^{-1}\right) \beta_{3}-\left(J_{c o m} \beta_{2}+\beta_{2}^{-1} J_{M 2}^{*}\right)\right] \ddot{\theta}_{M 2}} \\
& \quad+\left[\left(b_{c o m} \beta_{1}+b_{M 1}^{*} \beta_{1}^{-1}\right) \beta_{3}-\left(b_{c o m} \beta_{2}+\beta_{2}^{-1} b_{M 2}^{*}\right)\right] \dot{\theta}_{M 1} \\
& \quad=-\beta_{2}^{-1 M 2} \tau_{M 2}-\tau_{\text {load }} \ldots \ldots \ldots \ldots \ldots \ldots \ldots \ldots \ldots
\end{aligned}
$$

\section{Control}

3.1 Acceleration Based Control Very high stiffness is necessary to realize a proper position control and in contrast, a very low stiffness is required for the force control. Robustness in control is necessary to obtain high performance in motion control systems. Acceleration based control is a very good approach to obtain robust control systems as it provides uniformity of the motion control in the acceleration domain and the robustness against the external disturbances and system uncertainties ${ }^{(22)}$.

3.2 Disturbance Observer The Disturbance Observer (DOB) is used for the robust operation of the system by compensating the disturbances as shown in Fig. $8^{(20)}$. The DOB can estimate the external disturbances and system uncertainties such as parameter variations, coupling and loading effects. This estimated disturbance is fed forward to the system to compensate the disturbance. The total disturbance torque $\tau_{\text {dis }}$ can be calculated as,

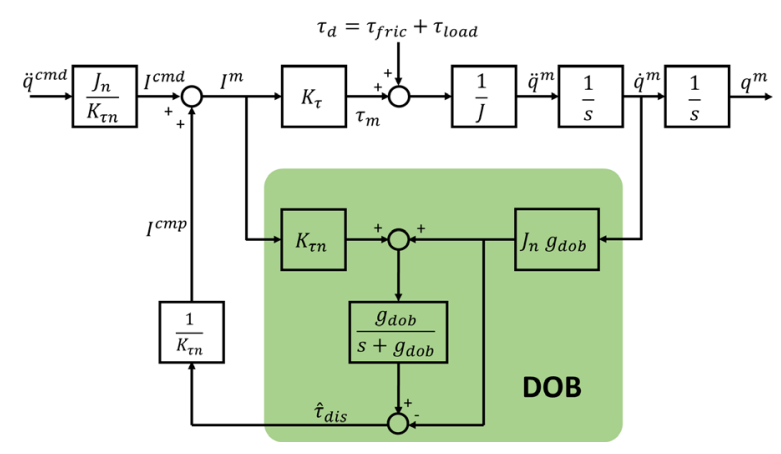

Fig. 8. Disturbance observer

$$
K_{\tau n} I^{m}-J_{n} \ddot{q}_{m}=\tau_{d i s}=\tau_{d}+\Delta J \ddot{q}_{m}-\Delta K_{\tau} I^{m} .
$$

Where, $K_{\tau}$ and $K_{\tau n}$ are the real and nominal torque coefficients and $I^{m}, J$ and $J_{n}$ are the motor current, real and nominal inertia respectively. $\Delta J=\left(J-J_{n}\right)$ and $\Delta K_{\tau}=\left(K_{\tau}-K_{\tau n}\right)$ are the inertia variation and the torque coefficient variation while $\tau_{d}=\left(\tau_{\text {fric }}+\tau_{\text {load }}\right)$ is the summation of friction and external disturbance respectively. Angle, velocity and acceleration of the motor is shown in $q^{m} \dot{q}^{m}$ and $\ddot{q}^{m}$ respectively. Disturbance is calculated from $I^{m}$ and $\dot{q}^{m}$ and fed through a first order low-pass filter with a cut-off frequency $g_{d o b}$ to calculate the estimated disturbance torque. Estimated disturbance $\hat{\tau}_{d i s}$ is given by,

$$
\hat{\tau}_{d i s}=\tau_{d i s} \frac{g_{d o b}}{s+g_{d o b}} .
$$

Equation (16) illustrates the possibility of precise disturbance compensation if it stays within the bandwidth of lowpass filter of the DOB. Open loop transfer function of Fig. 8 can be given as,

$$
\ddot{q}_{m}=\alpha \frac{s+g_{d o b}}{s+\alpha g_{d o b}} \ddot{q}^{c m d}-\frac{1}{J} \frac{s}{s+\alpha g_{d o b}} \tau_{d}, \cdots \ldots \ldots
$$

where, $\alpha=\frac{J_{n} K_{\tau}}{J K_{\tau n}}$ and $\frac{s}{s+\alpha g_{d o b}}$ is the sensitivity function of the inner loop ${ }^{(28)}$.

3.3 4-ch Bilateral Control There are several bilateral control methods ${ }^{(29)}$. The basic idea in the bilateral control is to transmit force information and position information between master robot and slave $\operatorname{robot}^{(30)}$. In this research, acceleration based four-channel bilateral control is used. Acceleration is taken as for the control reference so as to make it easier to control the two information, position and force at the same time.

Control targets of bilateral control system are achieved by position tracking between master robot $x_{m}$ and slave robot $x_{s}$ with the law of action and reaction between the forces of master and slave. These are represented in (3) and (4).

$$
\begin{aligned}
& x_{m}-x_{s}=0 \\
& F_{m}+F_{s}=0
\end{aligned}
$$

3.4 System Control The indices used in the rest of the paper and their meanings are shown in Table 2. For the controlling of the system, following transformation matrices were used. From the actuator space to the modal space the position tracking and the law of action and reaction is also to be realized. By considering the directions of torques and 
Table 2. Scripts used for concept modeling

\begin{tabular}{lll}
\hline \hline Script Type & Script Name & Meaning \\
\hline Superscript & $c m d$ & Command \\
Superscript & $r e f$ & Reference \\
Superscript & $r e s$ & Response \\
Subscript & $m$ & Master \\
Subscript & $s l$ & Slave-1 (Actuator space) \\
Subscript & $s 2$ & Slave-2 (Actuator space) \\
Subscript & $m, j o i n t$ & Master in joint space \\
Subscript & $s, j o i n t$ & Slave in joint space \\
Subscript & $s, t w i s t$ & Twist force of slave \\
Subscript & com & Common mode \\
Subscript & diff & Differential mode \\
Subscript & AJ & Actuator space to Joint space \\
Subscript & $J M$ & Joint space to Modal space \\
\hline \hline
\end{tabular}

angular position of Fig. 7, when both position and torque are in the same direction it will be the joint torque. When the torque and position are in the same directions, the torque will be twisting torque. Based on that, the transformation matrix $T_{A J}$ can be written as (21). Also the transformation between the modal space and the joint space has the twist control of the two gear systems. Therefore the transformation matrices can be written as (22).

$$
\begin{aligned}
& T_{A J}=\left[\begin{array}{cc}
1 & -1 \\
1 & 1
\end{array}\right] \\
& T_{J M}=\left[\begin{array}{cc}
1 & 1 \\
1 & -1
\end{array}\right]
\end{aligned}
$$

Here $T_{A J}$ denotes the transformation matrix between actuator space and the modal space. $T_{J M}$ denotes the transformation between modal space and joint space. $T_{A J}$ is used to transform the torque and the position responses of slaves to the corresponding modal space parameters as shown in (7). $T_{J M}$ is used to transform the modal space position and slave responses of master and slave actuators to obtain the joint space torque responses as shown in (8).

Therefore,

$$
\begin{aligned}
T_{A J}\left[\begin{array}{cc}
\tau_{s 1}^{r e s} & \theta_{s 1}^{r e s} \\
\tau_{s 2}^{r e s} & -\theta_{s 2}^{r e s}
\end{array}\right] & =\left[\begin{array}{cc}
1 & -1 \\
1 & 1
\end{array}\right]\left[\begin{array}{cc}
\tau_{s 1}^{\text {res }} & \theta_{s 1}^{\text {res }} \\
\tau_{s 2}^{\text {res }} & -\theta_{s 2}^{\text {res }}
\end{array}\right] \\
& =\left[\begin{array}{cc}
\tau_{s, \text { joint }}^{\text {res }} & * \\
\tau_{s, \text { twist }}^{\text {res }} & \theta_{s, \text { joint }}^{\text {res }}
\end{array}\right], \ldots \ldots \ldots \ldots \ldots
\end{aligned}
$$

and,

$$
\begin{aligned}
T_{J M}\left[\begin{array}{cc}
\tau_{m, j o i n t}^{\text {res }} & \theta_{m, j o i n t}^{\text {res }} \\
\tau_{s, \text { joint }}^{\text {res }} & \theta_{s, \text { joint }}^{\text {res }}
\end{array}\right] & =\left[\begin{array}{cc}
1 & 1 \\
1 & -1
\end{array}\right]\left[\begin{array}{cc}
\tau_{m, \text { joint }}^{\text {res }} & \theta_{m, \text { joint }}^{\text {res }} \\
\tau_{s, \text { joint }}^{\text {res }} & \theta_{s, \text { joint }}^{\text {res }}
\end{array}\right] \\
& =\left[\begin{array}{cc}
\tau_{\text {com }}^{\text {res }} & * \\
* & \theta_{\text {diff }}^{\text {res }}
\end{array}\right] . \ldots \ldots \ldots
\end{aligned}
$$

The twist control is achieved in the differential mode while the torque transmission for the bilateral control is achieved in the common mode. Angular Acceleration reference for the master manipulator in the joint space can be written as,

$$
\ddot{\theta}_{m, \text { joint }}^{r e f}=\frac{1}{2} C_{p}\left(\theta_{\text {diff }}^{\text {cmd }}-\theta_{\text {diff }}^{r e s}\right)+\frac{1}{2} C_{\tau}\left(\tau_{c o m}^{c m d}-\tau_{c o m}^{r e s}\right) .
$$

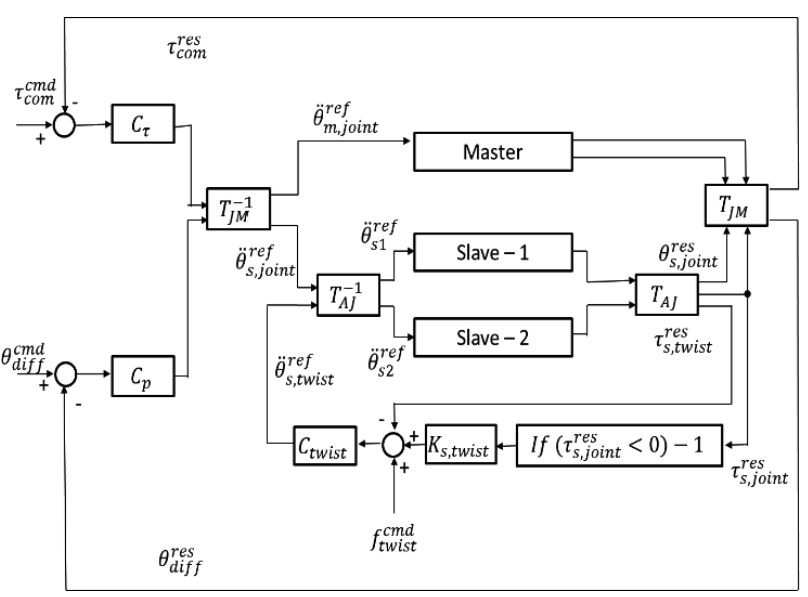

Fig. 9. Control block diagram

Here $C_{p}$ and $C_{\tau}$ denote position gain and torque gain respectively. $\theta_{d i f f}^{c m d}$ and $\tau_{c o m}^{c m d}$ are zero commands for the position of the differential mode and the torque in the common modes according to (3) and (4). For the slave manipulators,

$$
\begin{aligned}
\ddot{\theta}_{s 1}^{r e f}= & \frac{1}{2}\left[-\frac{1}{2} C_{p}\left(\theta_{\text {diff }}^{\text {cmd }}-\theta_{\text {diff }}^{r e s}\right)+\frac{1}{2} C_{\tau}\left(\tau_{\text {com }}^{\text {cmd }}-\tau_{\text {com }}^{r e s}\right)\right. \\
& \left.+C_{\text {twist }}\left(K_{s, t w i s t}\left|\tau_{s, j o i n t}^{r e s}\right|-\tau_{s, t w i s t}^{r e s}+\tau_{\text {twist }}^{\text {cmd }}\right)\right]
\end{aligned}
$$

and

$$
\begin{aligned}
\ddot{\theta}_{s 2}^{r e f}= & \frac{1}{2}\left[\frac{1}{2} C_{p}\left(\theta_{\text {diff }}^{\text {cmd }}-\theta_{\text {diff }}^{r e s}\right)-\frac{1}{2} C_{\tau}\left(\tau_{c o m}^{c m d}-\tau_{c o m}^{r e s}\right)\right. \\
& \left.+C_{\text {twist }}\left(K_{s, t w i s t}\left|\tau_{s, \text { joint }}^{\text {res }}\right|-\tau_{s, t w i s t}^{r e s}+\tau_{\text {twist }}^{c m d}\right)\right]
\end{aligned}
$$

Here, $C_{\text {twist }}$ is the twist constant and the effect of generated torque can be adjusted by changing $K_{s, t w i s t} . \tau_{\text {twist }}^{\text {cmd }}$ denotes twist command. In both (26) and (27) last terms are meant for the twist control. By using the twist control, a constant twisting torque is maintained in between the two slaves to cancel the backlash, as described in Sect. 2.3.

The control block diagram is shown in Fig. 9. Here, the master, slave- 1 and slave- 2 consist of the Disturbance Observer (DOB) and the Reaction Torque Observe (RTOB) inside. The $\tau_{s, j o i n t}^{\text {res }}$ value is multiplied by -1 if it is less than zero, to get the modulus value to the Twist control part of the controller.

Since the controller is based on acceleration, the transformed acceleration values from the modal space to the actuator space is sent as the inputs to the master and slaves through the inverse transformation matrix $T_{A J}^{-1}$. The outputs of the transformation matrix $T_{J M}$ are fed back to the position control part and the torque control part of the controller.

3.5 Analysis The nominalized block diagram of a slave motor 1 together with the coupling effects can be illustrated as shown in Fig. 10. Because of the presence of the disturbance observer, the effect of internal disturbances and the external disturbances will be cancelled off. Only the load torque will be estimated by the reaction torque observer.

It is possible to easily show the sensitivity of the system to the disturbance, since the sensitivity function is just the high 


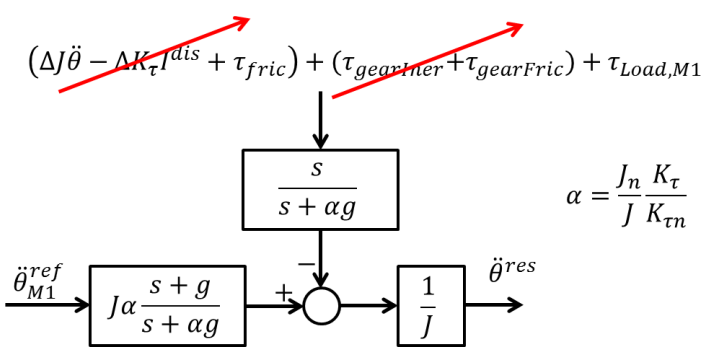

Fig. 10. Nominalized block diagram

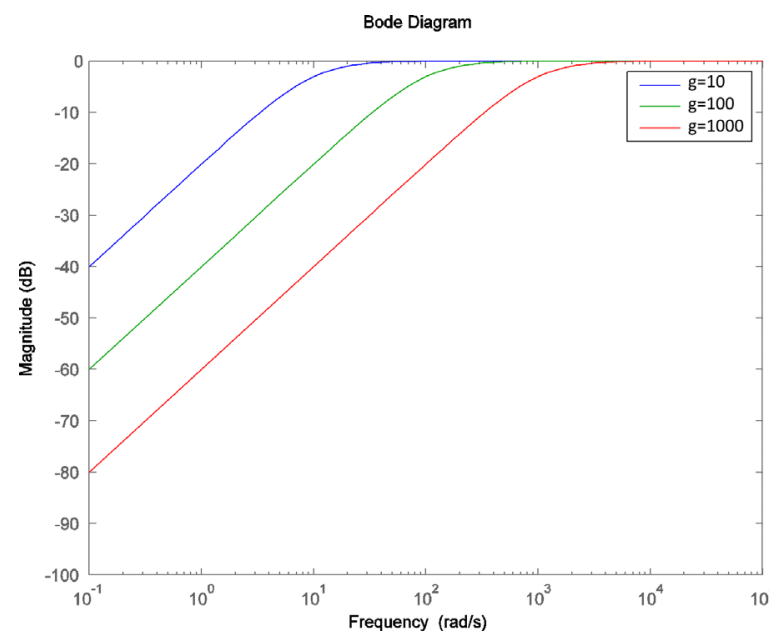

Fig. 11. Sensitivity of the system for external disturbance

pass filter of the nominalized model. Bode magnitude plots of different cutoff frequencies are shown in Fig. 11.

\section{Results and Discussion}

Experiments were carried out for both gear backlash cancellation and thrust wire backlash cancellation under the parameter values given in Table 3. For the gear backlash cancellation experiment, a planetary type gearbox having a speed reduction ratio of 43:1 was used on behalf of the cascaded gear arrangement. System assembly and the experimental setup for the gear experiment are shown in Fig. 12(a) and Fig. 13(a) respectively while Fig. 12(b) and Fig. 13(b) show those of the thrust wire experiment. The system assembly of the gear experiment consists of three encoder attached geared rotary motors with two actuators as slaves and the other as the master. The master actuator is used to interact with the end effector through bilateral control. The two slave geared motors are connected together in a way that they are coaxial as shown in Fig. 12(a). The thrust wire system assembly consists of three linear motors with two actuators as slaves. Here also, the master actuator is used to interact with the end effector. The two slave motors are connected to the two thrust wires which are clamped in parallel as shown in Fig. 12(b).

In the conventional method experimental case, one slave actuator is used. The experiments were carried out for both free motion and contact motion. For the thrust wire setup, the experiment was carried out for two cases as 0 degree case and 90 degree case with a bending radius of 300 millimeters. In all the experimental cases of both gear and thrust wire backlash cancellation, the master manipulator was moved in the periodic motion and observed the motion of the end effector
Table 3. Parameter values

\begin{tabular}{lll}
\hline \hline \multicolumn{1}{c}{ Parameter } & \multicolumn{1}{c}{ Gear case } & Thrust wire case \\
\hline Position feedback gain & $1600 \mathrm{~s}^{-2}$ & $1600 \mathrm{~s}-2$ \\
Velocity feedback gain & $80 \mathrm{~s}^{-1}$ & $80 \mathrm{~s}-1$ \\
Cut-off frequency of DOB & $80 \mathrm{rads}^{-1}$ & $500 \mathrm{rads}-1$ \\
Nominal mass & $0.5 \mathrm{~kg}^{-1}$ & $0.5 \mathrm{~kg}$ \\
Nominal motor constant & $0.5 \mathrm{NmA}^{-1}$ & $1.0 \mathrm{NA}-1$ \\
Constant torque/force & $0.05 \mathrm{Nm}^{2}$ & $2.0 \mathrm{~N}$ \\
\hline \hline
\end{tabular}

for the proposed method and the conventional method. The position responses of the master and slave and their errors are compared in the figures to show the reduction of the backlash errors of the proposed method. Also the corresponding torque/force responses obtained for the proposed method are shown to confirm the achievement of bilateral control between the master and the end effector along with the twist/thrust control.

4.1 Gear Backlash Cancellation Figure 14 shows the comparison of results obtained for the conventional method and the proposed method under the free motion while Fig. 15 shows the comparison of results obtained for the conventional method and the proposed method under the contact motion.

For the free motion case there was about 3 times reduction of the backlash on the proposed method over the conventional method. The backlash error was almost less than 0.1 radians in the proposed method while in the conventional case it was about 0.3 radians. The angular position tracking between the master and the end effector was almost well followed in the proposed method

The torque response graph of the free motion verifies the achievement of the twist control in the proposed method. The green and red lines are the torque responses of slaves and it can be seen that the response value has a limit from which the torque values of the two slaves do not go below to the zero level. This verifies that the two slaves always maintained a counteracting torque keep the whole gear system in a torsional state. Therefore the twist control is achieved. The blue line shows the torque response of the end effector, which is the summation of the two torque responses of two slaves. Pink line shows the torque response of the master, which is almost equal and opposite of the blue line, i.e. the end effector torque response. Therefore it validates the achievement of low of action and reaction.

Since the position tracking and the achievement of low of action and reaction are realized between the master and the end effector, the bilateral control is achieved in the free motion case.

In the contact motion there is a significant difference in the error in the above two instances. When the contact motion angular position responses are concerned for both conventional method and the proposed method under the contact motion in Fig. 15, it can be clearly seen the reduction of the error up to three times than that of the conventional case. In the conventional case the backlash is about 0.5 radians, while in the proposed method, it is about 0.15 radians. As in the case of free motion, the torque response figure corresponding to the contact motion consists of the force responses of two 

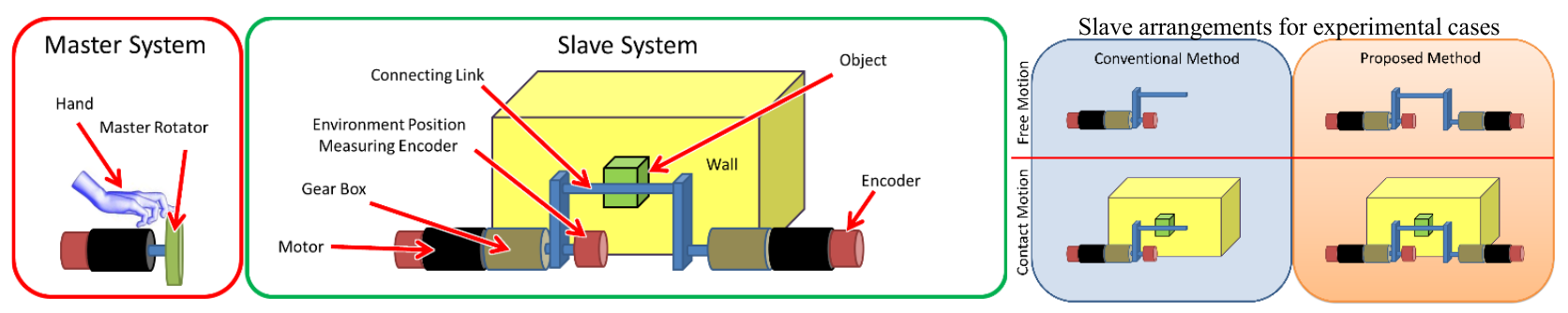

(a) Gear backlash cancellation

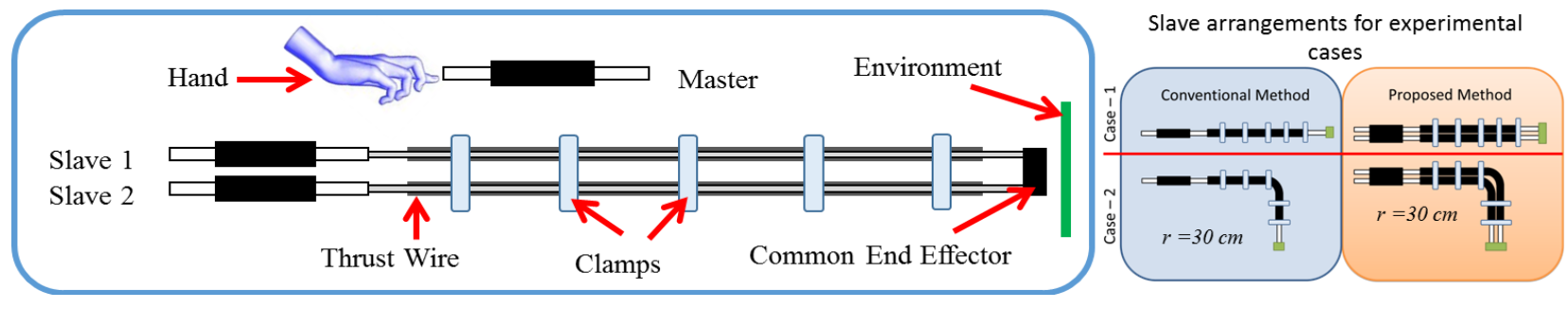

(b) Thrust wire backlash cancellation

Fig. 12. System assembly and experimental cases

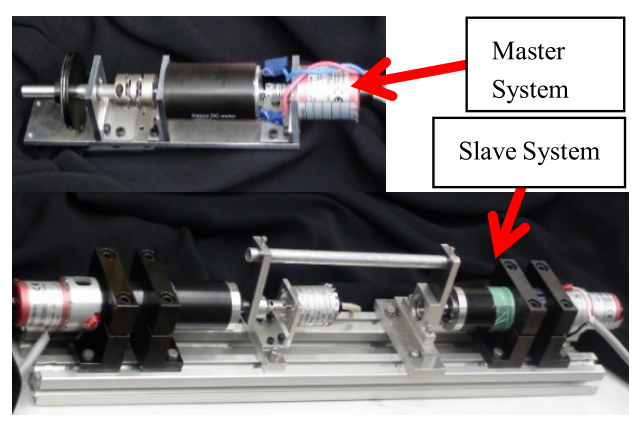

(a) Gear case

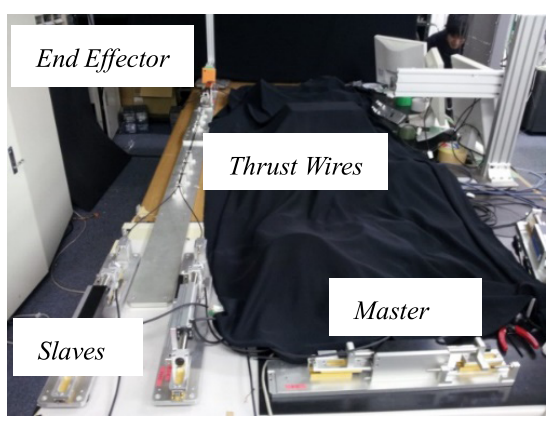

(b) Thrust wire case

Fig. 13. Experimental setups

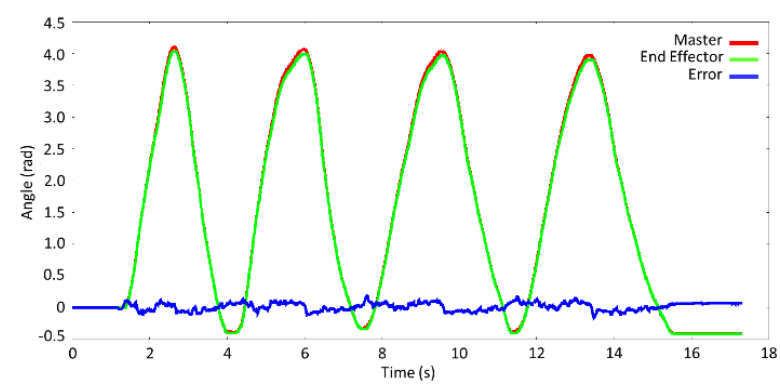

(a) Conventional method: position response

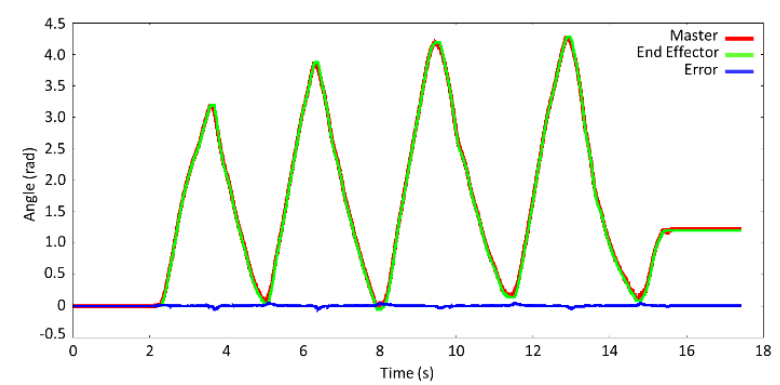

(b) Proposed method: position response

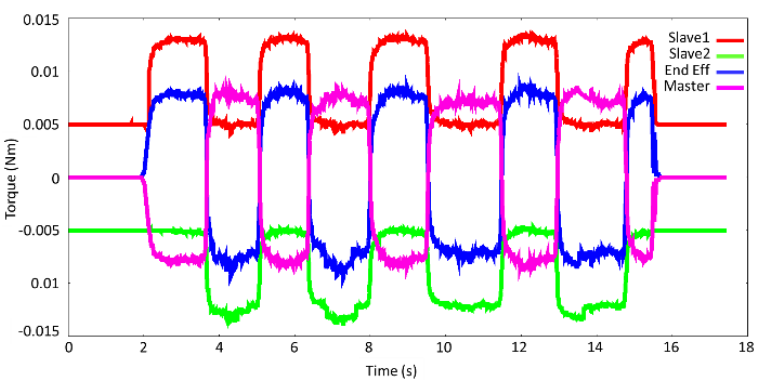

(c) Proposed method: torque response

Fig. 14. Experimental results of free motion

slaves, end effector and the master. As described in the free motion case, here in the contact motion also the achievement of the twist control and the low of action and reaction are realized.
As the position tracking and the achievement of low of action and reaction are realized between the master and the end effector in the contact motion also, the bilateral control is achieved. 


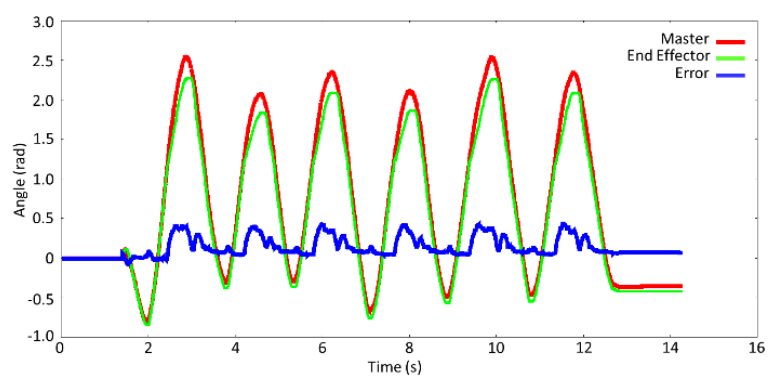

(a) Conventional method: position response

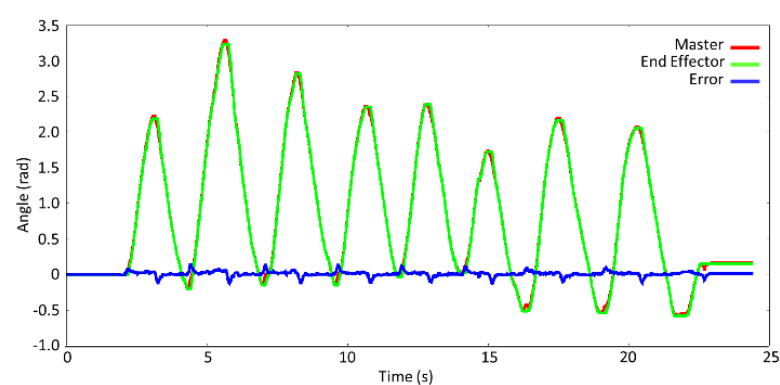

(b) Proposed method: position response

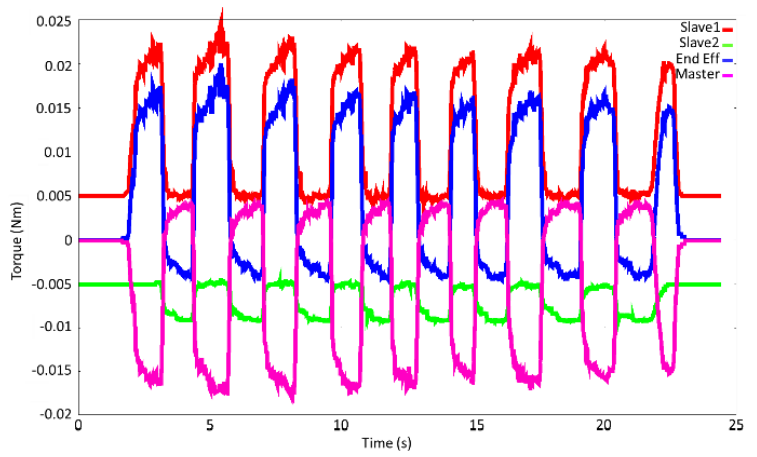

(c) Proposed method: torque response

Fig. 15. Experimental results of contact motion

Therefore it is evident that this proposed method contributes for the reduction of the backlash error up to three times than that of the conventional case in both free motion and contact motions under bilateral teleoperation.

4.2 Thrust Wire Backlash Cancellation The same control system that was used to cancel the backlash of the geared systems can also be used to cancel the backlash by changing the torques and the angular position to force and the position respectively for this case. It is possible to do so, due to the similarity of the modeling of the two systems and their concepts from the inception. Therefore, it is possible to solve the backlash problem of the thrust wires quite similar to the gear backlash cancellation with a unique control structure.

Figure 16 shows the comparison of results obtained for the conventional method and the proposed method, i.e. the 0 degree case while Fig. 17 shows the comparison of results obtained for the conventional method and the proposed method under the case 2, i.e. the 90 degree case.

Even under these cases, the responses of the position and force were considered for free motion and the contact motion. For the non-contact motion of both cases there was not any significant error difference in the backlash even with only the single thrust wire. The position tracking between the master and the end effector was almost well followed. This is because of the lower force acting against the movement of the inner wire even in the single wire instance. However if the error difference is carefully analyzed it can be seen that there is an improvement of about 4 times in the error reduction for the proposed method.

In the contact motion there is a significant difference in the error in the above two instances. When the contact motion position responses are concerned for both 0 degree case and the 90 degree cases in Fig. 16 and Fig. 17 respectively, it can be clearly seen the reduction of the error up to five times than that of the conventional case. Therefore it is evident that this proposed method contributes for the reduction of the backlash error up to five times in each case. Especially in Fig. 17, the increment of the error between the master and the end effector in the contact motion for the conventional method is clearly visible. Even at that instance the proposed method shows good position tracking, making the error to be a minimum.

When the force responses for the proposed method of master and two slaves in each case are considered, it is clear that the force values of the two slaves always manage a constant force value from which the force value does not go below. This verifies the achievement of the thrust control for this proposed method. Since the master response is equal and opposite to the summation of the force responses of the two slaves, it verifies the theory that was built for the proposal and also it verifies the achievement of bilateral control between the master and the end effector.

4.3 Experiments of Frequency Response As explained in the above sections, similar control structure was used to compensate the backlash of geared drives and the thrust wired systems. To evaluate the performance of the system, position and force responses under different frequencies were obtained by carrying out the experiments on thrust wire setup. Experiments were carried out by applying sinusoidal input signals having the frequencies of $0.5 \mathrm{~Hz}, 1 \mathrm{~Hz}$, $2 \mathrm{~Hz}, 3 \mathrm{~Hz}$ and $5 \mathrm{~Hz}$ under position control and force control. Transmissibility of the force and position tracking between the master and the end effector is considered to visualize and evaluate the performance of the system. Force applied on the system and the force observed at the end effector is plotted under the force control results given in Fig. 18. Also, for the position responses, the position of the master manipulator and the position of the end effector is plotted as in Fig. 19. The red lines show the corresponding parameter of the master 


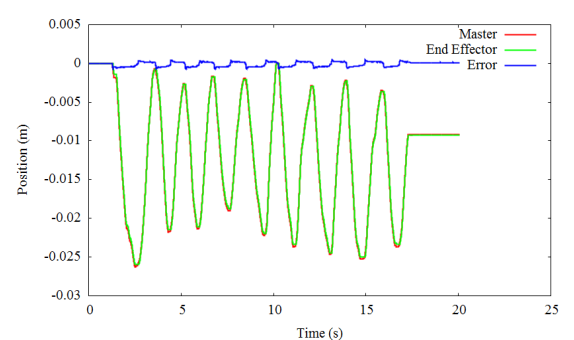

(a) Conventional method: free motion position response

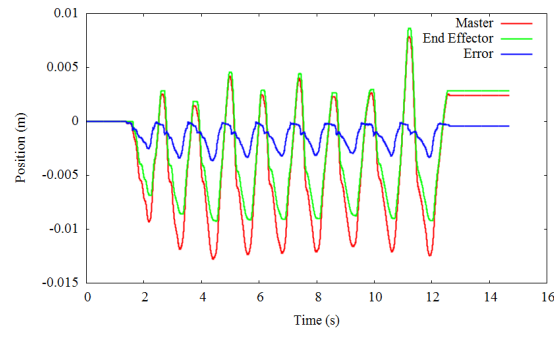

(d) Conventional method: contact motion position response

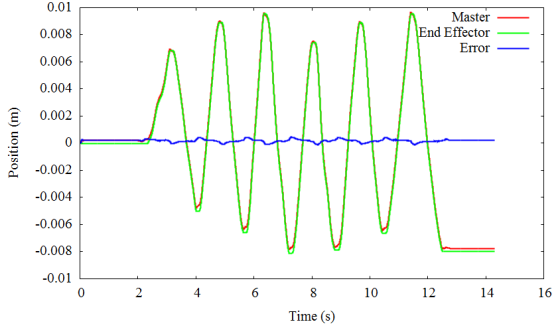

(b) Proposed method: free motionposition response

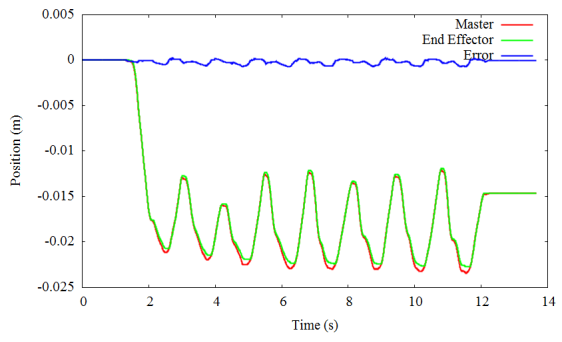

(e) Proposed method: contact motion position response

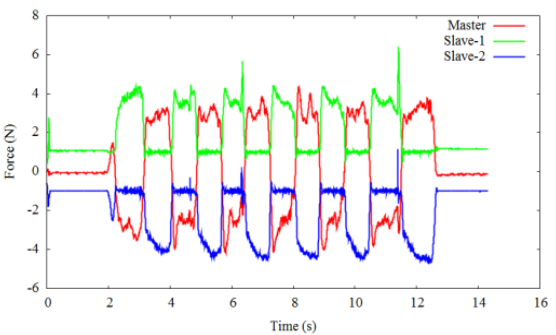

(c) Proposed method: free motion force response

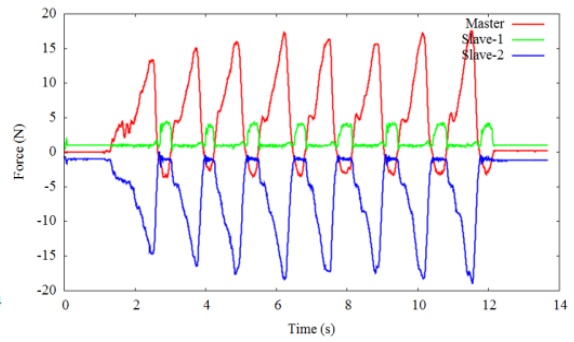

(f) Proposed method: contact motion force response

Fig. 16. Experimental results of 0 degree case

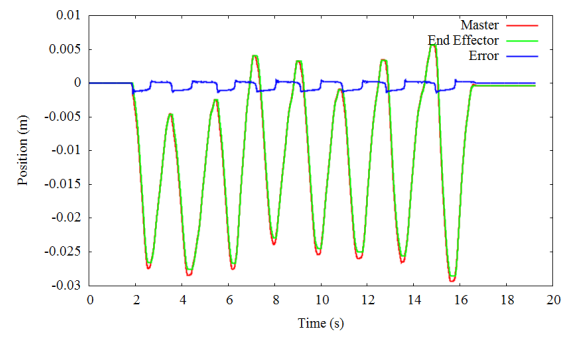

(a) Conventional method: free motion position response

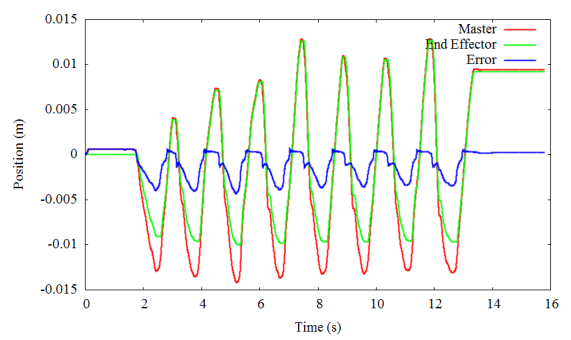

(d) Conventional method: contact motion position response

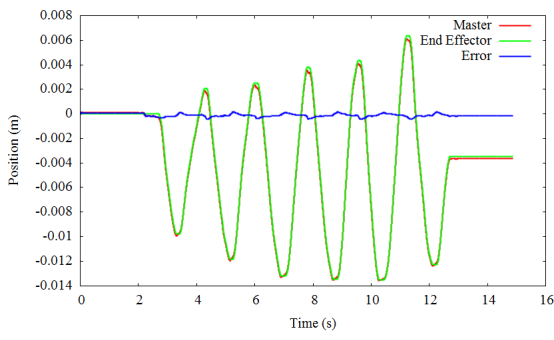

(b) Proposed method: free motion position response

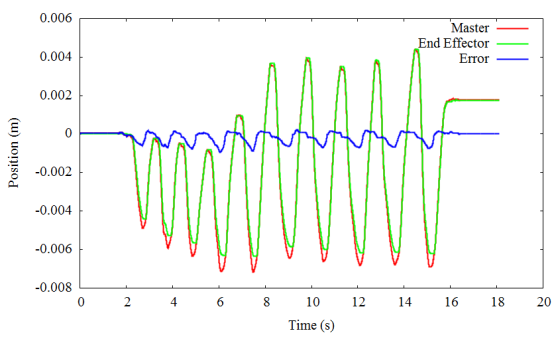

(e) Proposed method: contact motion position response

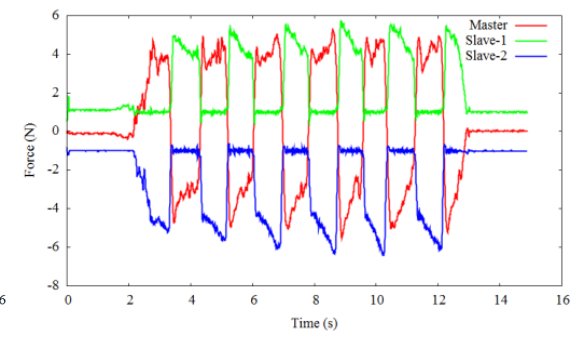

(c) Proposed method: free motion force response

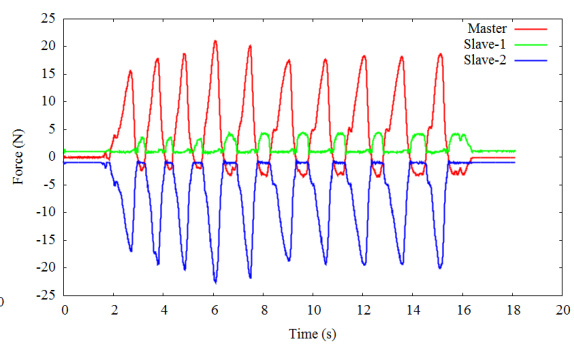

(f) Proposed method: contact motion force response

Fig. 17. Experimental results of 90 degree case

while the green lines show the corresponding parameter of the slave manipulator.

From the force control results, it can be seen that the results corresponding to the proposed method have better performance than that of the case where the backlash is presented. It is clearly seen that the glitch generated at the mechanical engagement inside the system is disappeared in the proposal. However still there is an error due to the non-linear frictional effects of the system which is not considered under the scope of this study.

Also the position control responses show very good position tracking between the master and the end effector. The error due to the backlash is almost negligible in the results obtained for the proposed method.

Therefore, it is clear that the proposed method guarantees the backlash compensation under different frequencies that enabling the system to show good performance in the haptic applications.

\section{Conclusion}

This paper proposed a novel backlash cancellation method that can be used to cancel the backlash presented in both geared drive systems and thrust wired systems. The backlash cancellation for both cases were achieved by a unique control method and the experiments have been carried out. This method does not use any additional force sensor or encoder at the end effector. Instead, this method uses two identical counter operating drives. These drives maintain the system in an interlocked state by applying equal and opposite torque/force and the load at the common end effector is 


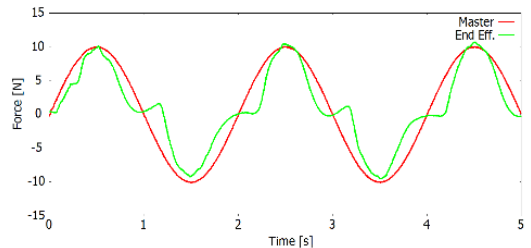

(a) Conventional method: $0.5 \mathrm{~Hz}$

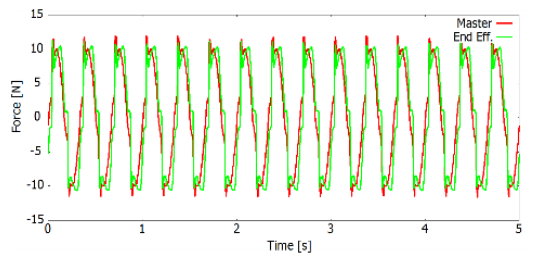

(d) Conventional method: $3 \mathrm{~Hz}$

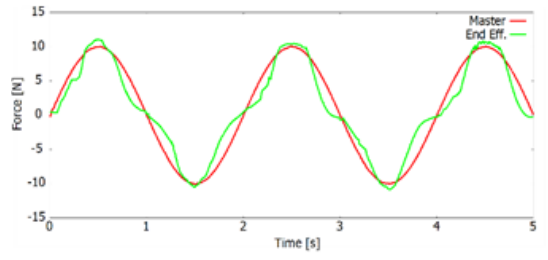

(f) Proposed method: $0.5 \mathrm{~Hz}$

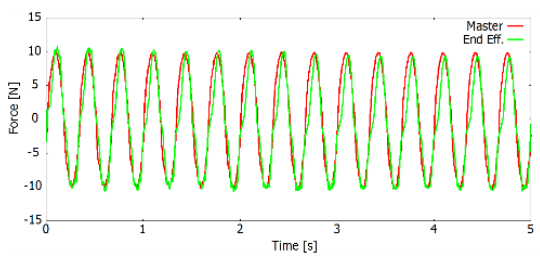

(i) Proposed method: $3 \mathrm{~Hz}$

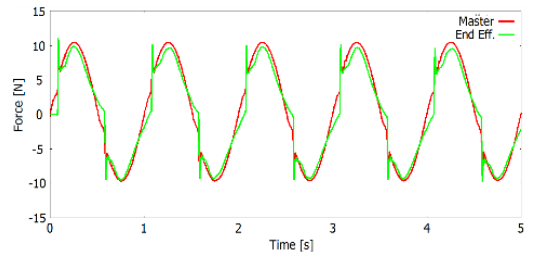

(b) Conventional method: $1 \mathrm{~Hz}$

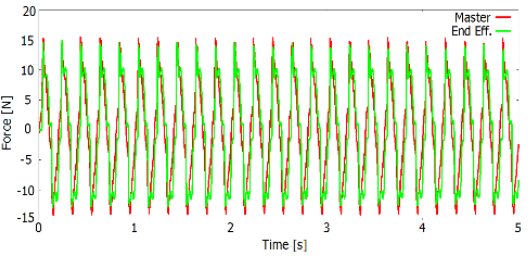

(e) Conventional method: $5 \mathrm{~Hz}$

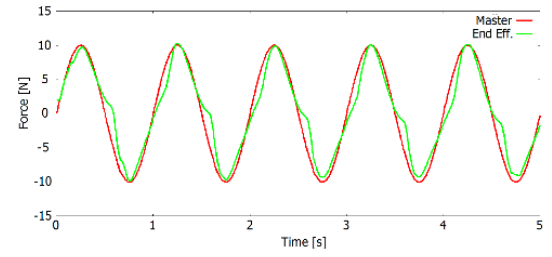

(g) Proposed method: $1 \mathrm{~Hz}$

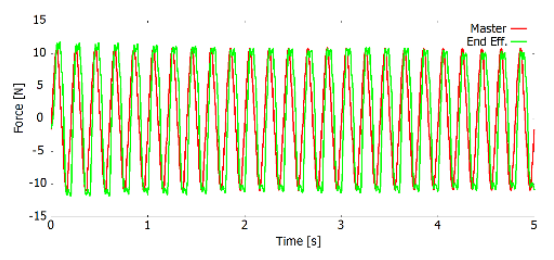

(j) Proposed method: $5 \mathrm{~Hz}$

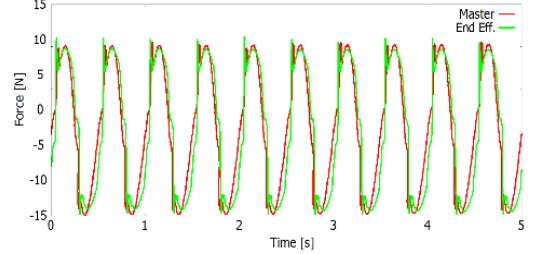

(c) Conventional method: $2 \mathrm{~Hz}$

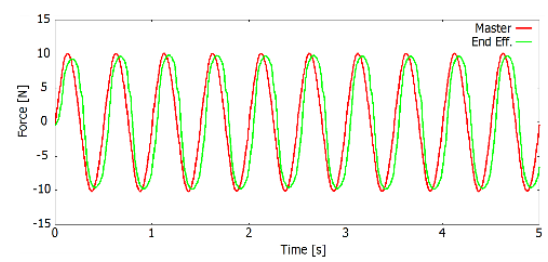

(h) Proposed method: $2 \mathrm{~Hz}$

Fig. 18. Force response results of different frequencies

shared by each actuator depend on the direction of operation. These two drives operate as slaves for the bilateral operation and the haptic transmission was taken place between the master manipulator and the common end effector. Results obtained by the proposed method for both gear case and thrust wire case are compared with the results of their conventional methods of operation. The results of the experiments witness the reduction of backlash that was uniquely achieved by the proposed control method in both cases. Also the experiments carried out for different frequencies ensures good performance of this proposed system for different frequencies. This method is advantageous in the teleoperation in which case, it is mandatory to have a backlash free motion transmission to ensure proper reproduction of haptic information at the master side.

\section{Acknowledgment}

This research was supported in part by the Ministry of Education, Culture, Sports, Science and Technology of Japan under Grant-in-Aid for Scientific Research (S), 25220903, 2013.

\section{References}

( 1 ) K. Daeinabi and M. Teshnehlab: "Industrial Arc Welding Robot Defect Tracking System in Automotive Industry", in Proc. International Conference on Mechatronics and Automation (ICMA), pp.3937-3941 (2007)

( 2 ) A.A. Timmermans, H.A. Seelen, R.D. Willmann, and H. Kingma: "Technology-assisted training of arm-hand skills in stroke: concepts on reacquisition of motor control and therapist guidelines for rehabilitation technology design”, Journal of Neuroeng. Rehabil., Vol.6, No.1 (2009)

( 3 ) S. Hyodo and K. Ohnishi: "A method for motion abstraction based on haptic information directionality and an application to haptic motion display system", IEEE Trans. Ind. Electron., Vol.56, No.5, pp.1356-1363 (2009)

( 4 ) S. Hyodo, Y. Soeda, and K. Ohnishi: "Verification of flexible actuator from position and force transfer characteristic and its application to bilateral teleoperation system", IEEE Trans. Ind. Electron., Vol.56, No.1, pp.36-42 (2009)

( 5 ) K. Sugawara, Y. Suzuki, D. Yashiro, and K. Ohnishi: "Performance evaluation of thrust wires for application to endoscopic forceps", in Proc. IEEE International Symposium on Industrial Electronics (ISIE), pp.2201-2206 (2011)

( 6 ) Y. Ling and G. Tao: "Numerical design and analysis of backlash compensation for a multivariable nonlinear tracking system", in Proc. American Control Conference, Vol.5, pp.3539-3543 (1999)

( 7 ) C. He, Y. Zhang, and M. Meng: "Backlash compensation by neural-network online learning", in Proc. IEEE International Symposium on Computational Intelligence in Robotics and Automation, pp.161-165 (2001)

( 8 ) D. Gebler and J. Holtz: "Identification and compensation of gear backlash without output position sensor in high-precision servo systems", in Proc. 24th Annual Conference of the IEEE Industrial Electronics Society (IECON '98), pp.662-666 (1998)

(9) N.J. Ahmad and F. Khorrami: "Adaptive control of systems with backlash hysteresis at the input", in Proc. American Control Conference, Vol.5, pp.3018-3022 (1999)

(10) I. Kolnik and G. Agranovich: "Backlash compensation for motion system with elastic transmission", in Proc. 27th Convention of Electrical \& Electronics Engineers in Israel (IEEEI), pp.1-5 (2012) 


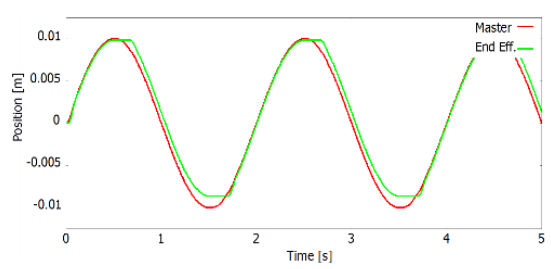

(a) Conventional method: $0.5 \mathrm{~Hz}$

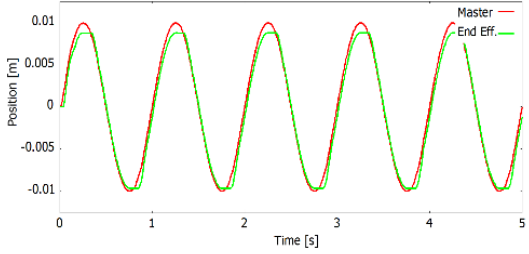

(b) Conventional method: $1 \mathrm{~Hz}$

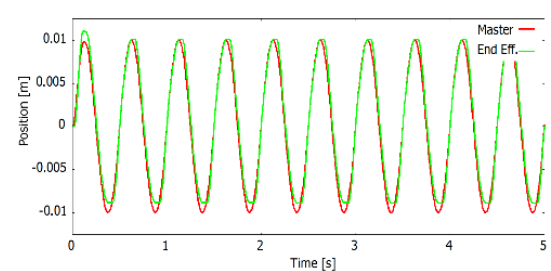

(c) Conventional method: $2 \mathrm{~Hz}$

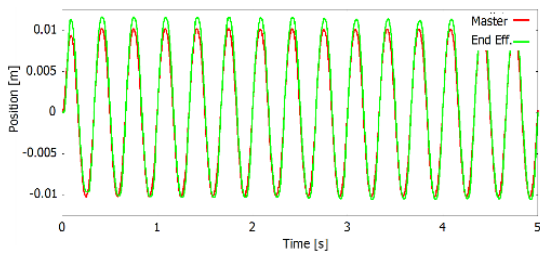

(d) Conventional method: $3 \mathrm{~Hz}$

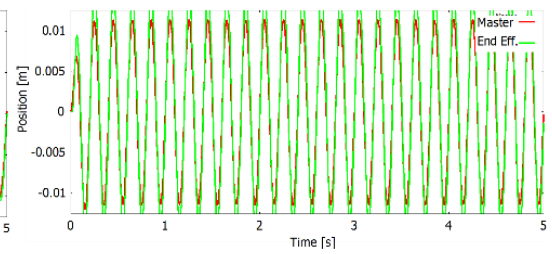

(e) Conventional method: $5 \mathrm{~Hz}$

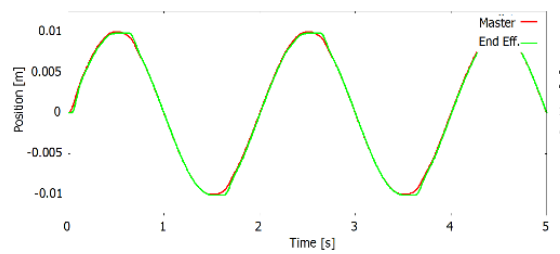

(f) Proposed method: $0.5 \mathrm{~Hz}$

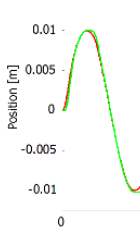

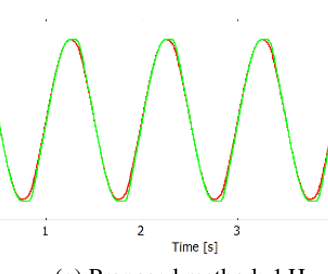

(g) Proposed method: $1 \mathrm{~Hz}$
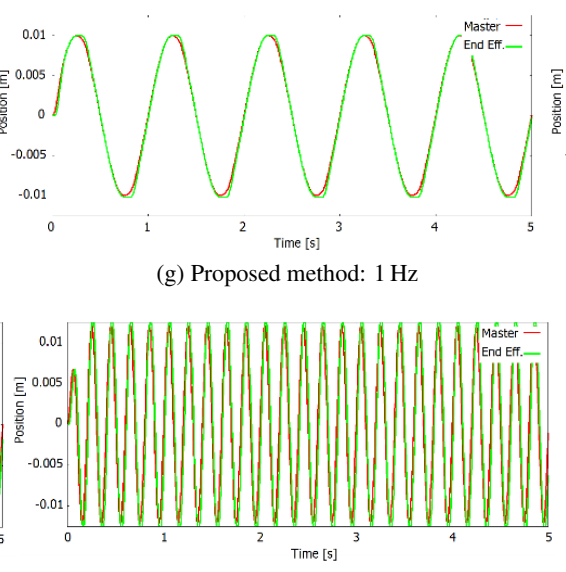

(j) Proposed method: $5 \mathrm{~Hz}$

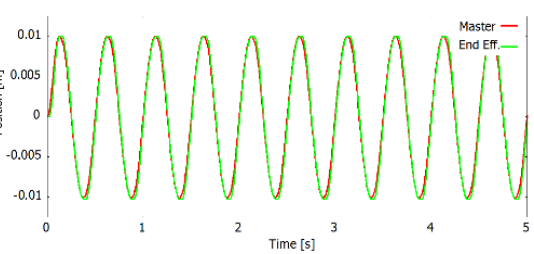

(h) Proposed method: $2 \mathrm{~Hz}$ (i) Proposed method: $3 \mathrm{~Hz}$

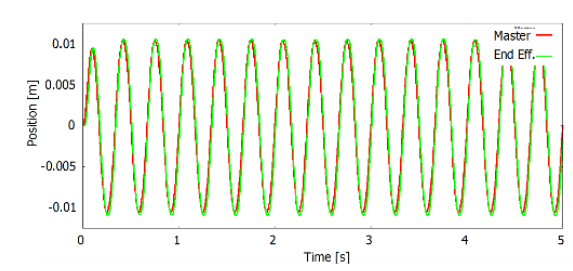

Fig. 19. Position response results of different frequencies

(11) B. Jung, J. Kong, B. Lee, S. Ahn, and J. Kim: "Backlash compensation for a humanoid robot using disturbance observer", in Proc. 30th Annual Conference of IEEE Industria 1 Electronics Society (IECON), Vol.3, pp.2142-2147 (2004)

(12) C. Kim, M. Kang, J. Kim, and U. Huh: "Implementation of disturbance observer for compensating backlash”, in Proc. ICCAS- SICE, 2009, pp.18121816 (2009)

(13) S.M. Shahruz: "Performance Enhancement of a Class of Nonlinear Systems by Disturbance Observers", IEEE/ASME Trans. on Mechatronics, Vol.5, No.3, pp.319-323 (2000)

(14) S.M. Shahruz, C. Cloel, and M. Tomizuka: "Suppression of Effects of Nonlinearities in a Class of Nonlinear Systems by Disturbance Observers", in Proc. American Control Conf., pp.2340-2345 (2002)

(15) Komoda, N. Maehii, and T. Han: "Control of Redundant Manipulators Considering Order of Disturbance Observer", in Proc. IEEE Trans. Ind. Elec., Vol.47, No.2, pp.413-419 (2000)

(16) M. Ito, Y. Komazawa, and T. Nishibori: "Multi-Drive Motor", in United States patent 2008/0150454 A1 (2008)

(17) S.L. Chang and L.W. Tsai: "On the redundant-drive backlash-free robotic mechanisms", Journal of Mechanical Design, Vol.115.2, pp.247-254 (1993)

(18) J. Jaisheela and K.S. Kumar: "Control of backlash in electromechanical system-design and simulation based approach", in Proc. International Conference on Devices, Circuits and Systems (ICDCS), pp.427-431 (2012)

(19) M. Odai and Y. Hori: "Speed control of two-Inertia System with Gear Backlash Based on Gear Torque Compensation", IEEJ Trans, Vol.134, No.2 (2001)

(20) M. Nordin and P.O. Gutman: "Controlling Mechanical Systems with Backlsh-a Survey”, Trans. of Automatica, Vol.38 (2002)

(21) R. Merzouki and J.C. Cadiou: "Estimation of backlash phenomenon in the electromechanicla actuator", Control Engineering Practice, Vol.13 (2005)

(22) K. Ohnishi, M. Shibata, and T. Murakami: "Motion control for advanced mechatronics", IEEE/ASME Trans. Mechatronics, Vol.1, No.1, pp.56-67 (1996)

(23) D.K. Prasanga, T. Mizoguchi, K. Tanida, and K. Ohnishi: "Compensation of Backlash for Teleoperated Geared Motor Drive Systems", in Proc. 39th Annual Conference of the IEEE Industrial Electronics Society (IECON), pp.4067-4072 (2013)

(24) C. Mitsantisuk, K. Ohishi, and S. Katsura: "Design of ball-cage based miniature stroke for integration in a flexible actuator with thrust wire", in Proc. 1st International Symposium on Access Spaces (ISAS), pp.59-64 (2011)

(25) M. Kawamoto, Y. Suzuki, D. Yashiro, and K. Ohnishi: "Estimation of disturbance force caused by thrust wire using a spring and damper model", in Proc. 36th Annual Conference on IEEE Industrial Electronics Society (IECON), pp.1263-1268 (2010)

(26) T. Nozaki, Y. Suzuki, and K. Ohnishi: "Transmission of Force Sensations by Hand of Multi-DOF Master-Slave Robot Using Tendon-Driven Mechanism”, IEEJ Trans. IA, Vol.131, No.3, pp.297-303 (2011)

(27) D.K. Prasanga, K. Tanida, T. Mizoguchi, and K. Ohnishi: "Evaluation of a Backlash Compensation Method Using Two Parallel Thrust Wires", in Proc. 22nd IEEE International Symposium on Industrial Electronics (ISIE), pp.1-6 (2013)

(28) E. Sariyildiz and K. Ohnishi: "A Guide to Design Disturbance Observer", J. Dyn. Sys. Meas. Control, Vol.136, No.2 (2013)

(29) W. Iida and K. Ohnishi: "Reproducibility and operationality in bilateral teleoperation", in Proc. The 8th IEEE International Workshop on A dvanced Motion Control (AMC'04), pp.217-222 (2004)

(30) T. Sato, S. Sakaino, and K. Ohnishi: "Methods for reduction of operational force in force sensor-less bilateral control with thrust wire", in Proc. 3rd Conf. on Human System Interactions (HSI), pp.412-418 (2010) 
D Kasun Prasanga (Non-member) received his bachelor's degree

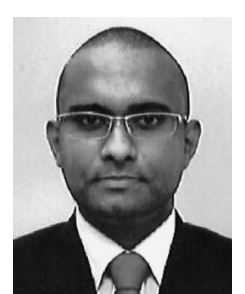
from University of Moratuwa, Sri Lanka and master's degree from Keio University, Japan. Currently he is a Ph.D. candidate in the School of Integrated Design Engineering, Keio University, Japan. His research interests include Haptics, Motion control, Biped locomotion and Robotics.

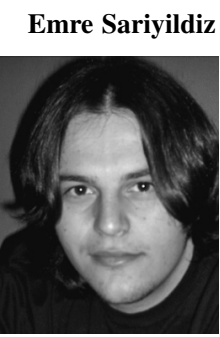

Kouhei Ohnishi (Fellow) received the B.E., M.E., and Ph.D. de-

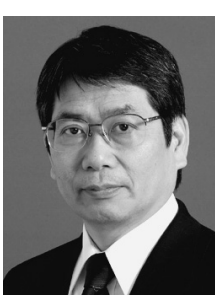
grees in electrical engineering from The University of Tokyo, Tokyo, Japan, in 1975, 1977, and 1980, respectively. Since 1980, he has been with Keio University, Yokohama, Japan. His research interests include mechatronics, motion control, robotics, and haptics. Dr. Ohnishi was the recipient of the Best Paper Awards from the Institute of Electrical Engineers of Japan and the Japan Society for Precision Engineering and the Outstanding Paper Awards at IECON ' 85, IECON '92, and IECON '93. He was also the recipient of the EPEPEMC Council Award and the Dr.-Ing. Eugene Mittelmann Achievement Award from the IEEE Industrial Electronics Society in 2004. 